\title{
Infections of cervical disc space after dental extractions
}

\author{
J. A. FEIGENBAUM AND W. E. STERN \\ From the Department of Surgery, Division of Neurosurgery, \\ UCLA School of Medicine, Los Angeles, California 90024, U.S.A.
}

SYNOPSIS Two patients with infections of the cervical intervertebral disc space after dental procedures carried out by the same oral surgeon exhibited similar clinical courses and radiographic appearances. Both had bacteriological confirmation of infection by needle aspiration and were treated with appropriate antibiotics and bracing of the neck. The presumed aetiology and the possible pathogenesis are described. Evidence suggests that the two infections were the result of needle injection of a contaminated solution, the organisms of which haematogenously lodged in the intervertebral discs in the cervical region. Lymph drainage from the gums and teeth is suggested as a possible route of inoculation.

Two patients recently developed signs suggestive of cervical disc space infection after dental procedures performed by the same oral surgeon. The temporal relationship of the dental work and the onset of the symptoms, together with the similarity of courses, suggested a cause and effect relationship.

\section{CASE 1}

A 17 year old girl entered the UCLA Hospital with severe pains of the neck and shoulder dating from a dental proeedure one month before. She had been given a general anaesthetic consisting of intravenous sodium methohexital for extraction of a tooth behind the left upper central incisor. The tooth had erupted in a normal direction but was removed because of its supernumerary status. No evidence of intraoral infection was seen at the time of the procedure, and local anaesthetic was employed at the site of the extraction only. After the procedure the patient noted a diffuse headache that persisted for approximately two hours. She had no discomfort until the next day when she bent over and noticed that severe pain in the posterior cervical region prevented her from completely flexing her neck. The pain radiated up to the occipital region and down to the midthoracic area in the mid-line. She was admitted to her community hospital where she was noted to have a limitation of neck movement and a temperature of $39.4^{\circ} \mathrm{C}\left(103^{\circ} \mathrm{F}\right)$. Lumbar puncture was normal with regard to pressure and cellular reaction in the cerebrospinal fluid. She remained in hospital for five days during which time she received intravenous antibiotics and improved slightly. Two weeks later she was rehospitalized for continued posterior neck pain and limitation of neck movement. Cervical traction increased her pain. Local anaesthetic infiltration of cervical muscular 'trigger' points also was unsuccessful. One month after the onset of symptoms, she was admitted to the UCLA Hospital where she described her pain as being constant, located in the posterior cervical and upper thoracic region, and radiating into the upper shoulders bilaterally. Movement of the neck aggravated the pain, but there was no radiation into the extremities. Postural change provided no help. There was no history of serious illness, infection, or neck trauma. Physical examination was unremarkable and showed her to be well-developed and normally proportioned for her age. Positive physical findings were confined to the neck and spine. She had tenderness and spasm of the paraspinous cervical musculature, and her neck movements were limited in all directions. There were no palpable spinous deformities, and there was no motor weakness, sensory abnormality, or reflex change. She showed a fine tremor of the outstretched arms, but her coordination was normal. Her gait, although guarded, was steady. The straight-leg raising manoeuvre was performed to $90^{\circ}$ bilaterally, and there were no other signs of meningeal irritation.

Laboratory studies disclosed the following values: white blood cell count (WBC), $12,200 / \mathrm{mm}^{3}$ with $83 \%$ segmented neutrophils, $11 \%$ lymphocytes, $4 \%$ monocytes, and $2 \%$ eosinophils; haemoglobin, $12 \cdot 1$ $\mathrm{g} / 100 \mathrm{ml}$; haematocrit reading, $35.5 \%$; fasting blood 

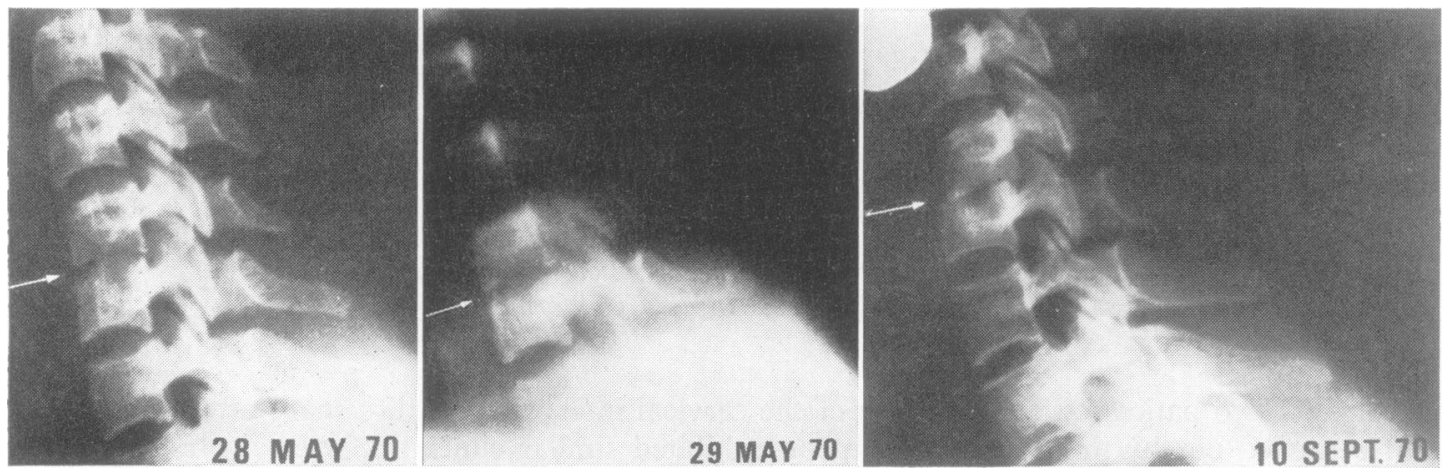

FIG. 1 (left). Case 1. Lateral cervical spine radiograph showing loss of the disc space and lucencies in the vertebra at C5/6. FIG. 2 (centre). Case 1. Midline sagittal tomograph of cervical spine showing involvement of C5/6 disc and adjacent vertebra. FIG. 3 (right). Case 1. Follow-up radiograph of cervical spine showing some displacement at C5/6.

sugar, $83 \mathrm{mg} / 100 \mathrm{ml}$; sedimentation rate, $41 \mathrm{~mm} / \mathrm{hr}$. Lumbar puncture revealed an opening pressure of $100 \mathrm{~mm}$ water. The fluid was clear and colourless with 3 white cells $/ \mathrm{mm}^{3}$ and a protein of $31 \mathrm{mg} / 100$ $\mathrm{ml}$. Cerebrospinal fluid (CSF) glucose was $83 \mathrm{mg} /$ $100 \mathrm{ml}$. Radiographs of the cervical spine revealed swelling of the prevertebral soft tissues at the C4/5 and C5/6 levels. There was a loss of the disc space at C5/6 with some loss of the superior margin of the C6 vertebral body and lucency in the inferior aspect of the body of C5 vertebra (Fig. 1). Cervical tomographs confirmed the lytic defect at the $\mathrm{C} 5 / 6$ intervertebral space involving the lower margin of $\mathrm{C} 5$ and the upper margin of $\mathrm{C} 6$ vertebrae (Fig. 2). Aspirate was taken from the disc space under fluoroscopic control with a gauge 18 needle inserted between the carotid sheath and trachea into the intervertebral disc. The culture grew an enterobacter species. The patient was given kanamycin sulphate (320 mg twice daily) for 10 days and then chloramphenicol $(500 \mathrm{mg}$ every six hours) for an additional four weeks. She was placed in a four-poster neck brace and was able to walk without discomfort by the time of her discharge six weeks after the onset of symptoms. Follow-up radiographs taken at discharge showed some further collapse of the disc space and a slight anterior dislocation of C6 vertebra. However, when studied $4 \frac{1}{2}$ months after the onset of illness, the lytic process had not progressed (Fig. 3).

\section{CASE 2}

A 38 year old woman entered the UCLA Hospital with severe posterior neck pain of one month's duration. She had undergone general anaesthesia with sodium methohexital for extraction of a painfue right mandibular second molar and a broken upperen left first bicuspid tooth. There was no gingival reac tion at the time of extraction. Local anaesthesia was used in addition to the methohexital for the extract. tion of both teeth. When the patient awakened from the anaesthesia, she experienced generalized shaking $\rightarrow$ chills that persisted through the evening. The nexk morning her neck was stiff and painful with move 0 ment. Symptoms increased, movements became more restricted, and pain persisted at rest. She was adê⿳亠口冋 mitted to a community hospital and remained for seven days in cervical traction that exacerbated her symptoms. The pain did not radiate into the upper extremities, and she had no weakness or sensory symptoms. She had no history of systemic infections or accidents or injuries involving her cervical spine. The severe cervical pain during her fourth week of illness led to her referral to the UCLA Hospital one month after the onset of her trouble.

At physical examination, she held her neck stiffly and guarded any attempts to manipulate it. Pain was accentuated by any movement, including vertical compression of the cervical spine. The cervical paraspinous musculature was in spasm and tender from the suboccipital region to the level of the T3 spinc. There was no weakness of the upper or lower extremities. Sensory examination and reflexes were normal, as was the remainder of her examination.

Laboratory findings showed the following values: WBC, 5,100 per $\mathrm{mm}^{3}$ with $60 \%$ segmented neutrophils, $29 \%$ lymphocytes, $9 \%$ monocytes, $1 \%$ bands, and $1 \%$ eosinophils; haemoglobin $12.3 \mathrm{~g} / 100 \mathrm{ml}$; haematocrit reading, $36.5 \%$; corrected sedimentation rate, $39 \mathrm{~mm} / \mathrm{hr}$. Lumbar puncture revealed an 

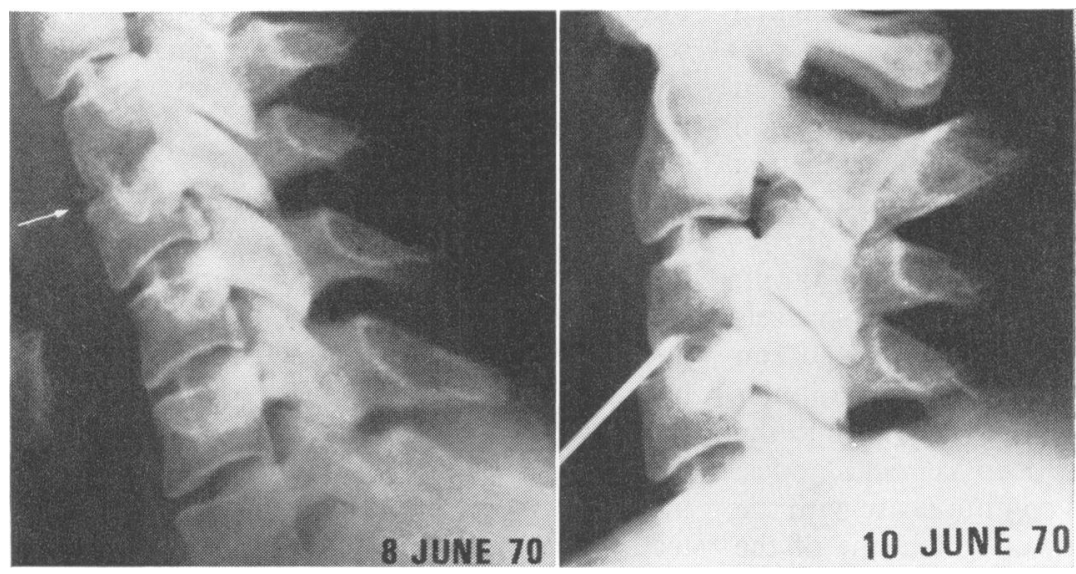

FIG. 4 (left). Case 2.

Radiograph of cervical spine showing destruction of disc space at $C 3 / 4$.

FIG. 5 (right). Case 2. Radiograph taken during percutaneous aspiration of $\mathrm{C} 3 / 4$ disc space.

opening pressure of $150 \mathrm{~mm}$ water. The fluid was clear and colourless, and no white cells were present. The CSF protein was $71.7 \mathrm{mg} / 100 \mathrm{ml}$, and the sugar was $66 \mathrm{mg} / 100 \mathrm{ml}$. The culture was negative. Radiographs of the cervical spine showed a collapse of the C3/4 disc space with some minimal destruction of the inferior aspect of the body of $\mathrm{C} 3$ and the superior portion of the body of $\mathrm{C} 4$ vertebra. There was an anterior subluxation of $\mathrm{C} 4$ upon $\mathrm{C} 3$ vertebra of $3 \mathrm{~mm}$ (Fig. 4). The day after admission, a needle aspiration of the $\mathrm{C} 3 / 4$ space was performed following the method described in case 1 (Fig. 5), and the culture grew an enterobacter species. Initially, kanamycin sulphate (300 mg every 12 hours) was given, but, based upon the tube dilution studies of the enterobacter, gentamicin sulphate $(50 \mathrm{mg}$ every 12 hours) was substituted for a two-week course. She was discharged free of neck discomfort two weeks after admission with a four-poster brace for use when in the upright position. Follow-up examination revealed no recurrence of neck pain and no evidence of neurological impairment. Repeat radiographic study demonstrated continued collapse of the C3/4 interspace and minimal subluxation. She was lost to follow-up five months after the onset of her illness.

\section{DISCUSSION}

PATHOGENESIS Although most attention in the literature has been directed toward infections of the disc spaces resulting from intervertebral disc operations (Turnbull, 1953; Stern and Crandall, 1959; Mella, 1965), the cases described herein caused us to focus our attention on the problem of interspace infection introduced by needle injection. The procedure of discography, whereby a needle is introduced directly into the intervertebral disc to facilitate diagnosis of disc disease, is a common source of infection. This complication has been discussed by De Séze and Levernieux (1952) who reviewed 13 cases of an inflammatory response after $50 \%$ to $70 \%$ diodon polyvidone was used for discography. Another possible route of infection is the accidental puncture of the disc space while performing a lumbar puncture in the presence of a bacterial meningitis. Less obvious and more indirect routes of infection have been reported by numerous authors. Eliason (1965) reported two cases of disc and adjacent vertebral body infection after needle biopsy of the prostate gland. The presumed route of infection was through the veins of Batson's plexus from the prostate to the anterior borders of the lumbar vertebral canal. Cervical osteomyelitis after the intravenous injection of contaminated succinyl choline chloride during induction of anaesthesia was reported by Del Toro et al. (1970). The common organism recovered from blood culture among their patients was Serratia marcescens. Six of their patients showed radiological evidence of infection of the cervical vertebral body. These authors mentioned hyperextension of the neck during intubation as a possible initial cause of injury to the vertebral column. The occurrence of vertebral body osteomyelitis among those who indulge in narcotics with contaminated techniques of 
intravenous administration is well documented (Bryan et al., 1973).

If our cases do, indeed, signify contamination of the vertebral discs in the cervical region through needle injection of local anaesthetics, we must consider the most probable route. The lymph drainage from the gums and teeth deserves consideration (Grant, 1958; Pernkopf, 1963). From the upper teeth, the lymph vessels pass through the infraorbital foramen and run with the facial artery to the submandibular nodes. Those of the lower teeth run through the mandibular canal to the deep cervical nodes. The submandibular nodes communicate with the upper deep cervical nodes, of which one of the more notable is the jugulodigastric node. This structure lies below the posterior belly of the digastric muscle where the common facial vein enters the internal jugular vein. Lymphatics from the jugulodigastric node are believed to go to prevertebral nodes, some of which lie in the alar space immediately in front of the prevertebral fascia. There is, therefore, a possible, albeit circuitous, route for contaminated material to be carried from the gums or the teeth through the lymphatic systems to the prevertebral tissues. In the two cases reviewed here, however, this would be an unlikely explanation in view of the prompt onset of symptoms of infection of the spine after the presumed inoculation.

The venous drainage of the maxilla and mandible is quite rich, and the development of intracranial infections resulting from cavernous sinus thrombosis has been well recognized after dental extractions (Haymaker, 1945). Intracranial abscesses have also been reported by Hollin et al. (1967) as the result of odontogenic infection. In their extensive review of the literature, however, these authors failed to note any intervertebral disc infections as a result of intra-oral disease.

The vertebral venous sytem has been well demonstrated by the injection techniques of Batson (1940). It is a valveless system, and it does appear conceivable that material injected into the veins of the upper or lower gums could enter into Batson's plexus of the cervical region without involving the superior vena cava system. In his study of retropharyngeal abscess, Grodinsky (1939) noted the possibility of spread of infection from the veins of the pharynx to the internal jugular veins with thrombosis and secondary extension to the retropharyngeal spaces. Another pathway for the spread of infection in the head and neck is through the various spaces delineated by the fascial planes. In his discussion of deep neck infections, Beck (1947) described a route of contamination from the infected bodies of the cervical vertebrae through the prevertebral fascia into the pharyngomaxillary space. He also noted that infections had been seen along the pathway of a needle placed to perform a mandibular nerve block. In those instances, the infection involved the pharnygomaxillary space. In addition, infections from the teeth of the maxilla, especially on the labial side, might result in extension into the sphenomaxillary fissure and the pharyngomaxillary fossa. Had a mandibular nerve block been administered to the patient described in our case 2 , this might conceivably have represented a combination of the events such as described by Beck leading to the contamination of the pre vertebral tissue. However, since the injections $s^{0} \vec{\omega}$ were local, in the gums immediately adjacent to the teeth removed, this does not seem likely $\frac{\text { क }}{2} 9$ Although a review of the recent dental and oraf $\vec{D}$ surgery literature failed to reveal a similar case Fein et al. (1969) reported a disc space infectiog at $\mathrm{C6} / 7$ that began approximately three weeks after an open comminuted fracture of the lef body of the mandible. The case was complicated by osteomyelitis of the mandible, and aspiration of the disc space failed to produce a positive culture, possibly because the patient had been treated with penicillin for 10 days before the attempt. This author noted the possible routes of infection as being direct extension via fascial planes, direct venous or lymphatic spread, or systemic haematogenous dissemination.

If our patients suffered disc space infections secondary to haematogenous spread, then we must note that the organism, 'enterobacter' is not a usual oral inhabitant (a nasopharyngeal culture of case 1 did return a few colonies of Escherichia coli). Bacteraemias after dental manipulation have been well recognized. Mitchell and Helman (1953). have reviewed the role of peridontal infections in systemic infectious disease. They found an incidence as high as $34 \%$ of bacteraemia resulting from standardized dental manipulations in patients without clinic- 
ally significant oral infections. With known periodontal disease, the bacteraemia rate more than doubled. Although the organisms reported are usually Gram positive cocci, a recent report by Goldberg (1968) described a case of bacteraemia after tooth extraction in which a blood culture grew Pseudomonas. With broad-spectrum antibiotics, the normal oral flora may well be changed to favour potent Gram negative organisms. Although our evidence is inconclusive, it seems most probable that the two infections in this report resulted from the injection of contaminated solutions, the organisms of which were haematogenously lodged in the vertebral column.

DIAGNOSIS AND THERAPY Severe pain and immobility of the affected portion of the spine, together with systemic symptoms or signs of inflammation, should suggest infection in the spinal column (Fischer, 1959). The blood should be cultured in the presence of an acute febrile response. The sedimentation rate, elevated in both of our patients, is a simple procedure that should be used as a part of the screening evaluation in similar clinical presentations. Laminograms help to delineate the disease process and to distinguish it from neoplastic conditions. A specific diagnosis of the offending agent may be made by a relatively innocuous needle aspiration of the disc space. Bed rest appears to be indicated during the period of systemic infection. Specific antibiotic therapy aids in the prompt resolution of symptoms as well as in combating the primary pathogen. In our second patient, the antibiotic agent was modified as a result of tube dilution studies performed on the enterobacter species, a valuable adjunct to specific therapy that is based upon the cultures from disc space aspiration. Immobilization of the infected cervical spine is required until a stable fusion develops. Although it has been charged by some to interfere with internal fixation in such cases, our experience with similar problems is that a firm union will occur within a matter of months, and external bracing is well tolerated until that is achieved. This is in agreement with the majority of authors who have discussed disc space infections. The relatively benign clinical courses of our two patients is also in accord with the usual course. The numerous features of our cases are consistent with certain of the cases described by Stern and Crandall (1959) after lumbar intervertebral disc operations. The outlook appears favourable.

\section{REFERENCES}

Batson, O. V. (1940). The function of the vertebral veins and their role in the spread of metastases. Annals of Surgery, 112, 138-149.

Beck, A. L. (1947). Deep neck infection. Annals of Otology, Rhinology, and Laryngology, 56, 439-481.

Bryan, V., Franks, L., and Torres, H. (1973). Pseudomonas aeruginosa cervical diskitis with chrondro-osteomyelitis in an intravenous drug abuser. Surgical Neurology, 1, 142144.

Del Toro, R. A., Rullan, J. A., Torregrosa, M., Lugo, A., and Freyre, J. L. (1970). Serratia marcescens septicemia with osteomyelitis of the cervical spine: an unusual epidemic. (Abstract.) Annals of Internal Medicine, 72, 796.

Eliason, O., and Dunlap, D. (1965). Osteomyelitis of the spine following needle biopsy of the prostate. Journal of Urology, 94, 271-275.

Fein, S. J., Torg, J. S., Mohnac, A. M., and Magsamen, B. F. (1969). Infection of the cervical spine associated with a fracture of the mandible. Journal of Oral Surgery, 27, 145149.

Fischer, K. A. (1959). Osteomyelitis and disc space infections of the spine. Journal of the Kentucky Medical Association, 57, 1066-1069.

Goldberg, M. H. (1968). Gram-negative bacteremia after dental extraction. Journal of Oral Surgery, 26, 180-181.

Grant, J. C. B. (1958). A Method of Anatomy, 6th edn, pp 793-796. Williams and Wilkins: Baltimore.

Grodinsky, M. (1939). Retropharyngeal and lateral pharyngeal abscesses: an anatomic and clinical study. Annals of Surgery, 110, 177-199.

Haymaker, W. (1945). Fatal infections of the central nervous system and meninges after tooth extraction. American Journal of Orthodontics, 31, 117-188.

Hollin, S. A., Hayashi, H., and Gross, S. W. (1967). Intracranial abscesses of odontogenic origin. Oral Surgery, 23, 277-293.

Mella, B. (1965). Inflammatory spondylitis. Journal of Neurosurgery, 22, 393-396.

Mitchell, D. F., and Helman, E. Z. (1953). The role of periodontal foci of infection in systemic disease: an evaluation of the literature. Journal of the American Dental Association, 46, 32-53.

Pernkopf, E. (1963). Atlas of Topographical and Applied Human Anatomy, vol. 1, pp. 227, 246, 247, and 249. Saunders: Philadelphia.

Séze, S. de, and Levernieux, J. (1952). Les accidents de la discographie. Revue du Rhumatisme et des Maladies Ostéo-articulaires, 19, 1027-1033.

Stern, W. E., and Crandall, P. H. (1959). Inflammatory intervertebral disc disease as a complication of the operative treatment of lumbar herniations. Journal of Neurosurgery, 16, 261-276.

Turnbull, F. (1953). Postoperative inflammatory disease of lumbar discs. Journal of Neurosurgery, 10, 469-473. 